\title{
Student management with HISinOne - A status report
}

\author{
Daniel Biella ${ }^{1}$, Uwe Blotevogel ${ }^{2}$, Malte $\mathrm{Ney}^{3}$, Sven Radermacher ${ }^{4}$ \\ ${ }^{1-4}$ University Duisburg-Essen, Schützenbahn 70, 45127 Essen, \{firstname.lastname\}@uni-due.de \\ Keywords \\ HEI administrative software, student management, HISinOne, STU, FIA, campus management.
}

\section{ABSTRACT}

The latest software generation by the German IT company HIS is named HISinOne. HISinOne is a centralized integrated web-browser based application with various functions supporting the student lifecycle in HEls. The University Duisburg-Essen (UDE) is one of the early adopters of this new software generation. In Q4/2012, the modules for student (STU) and student fee (FIA) administration have been rolled out successfully, running in an integrated IT-system, together with the exam management (EXA) and application (APP) module. This paper reports about the technological experiences made.

\section{BACKGROUND}

In 2008, the representatives of both the UDE and HIS GmbH signed a software piloting agreement for the next software generation HISinOne. By then, HIS had already taken the decision to migrate its existing HEl software portfolio to a new, Java-based, web-based software framework, called HISinOne. The new centralized approach also implied a complete and normalized redesign of the entire data model. The UDE - with its more than 30,000 enrolled students - has a long history in using HIS HEl software products and, therefore, the decision was made to participate in the challenge of migrating the four existing HIS application systems SOS, POS ZUL, and LSF into one modern web-based client/server application.

As one of the ten largest universities in terms of enrolled students, the UDE is using the new software solution for its 39,000 enrolled students and its 470 professors. At the EUNIS 2012 conference we presented our experience with the roll-out of the HISinOne module "EXA" for exam management. Now, one year later, the IT-system made a further step towards an integrated campus management system by migrating the student management from HIS-GX rich client applications to the centralized web-based front-end.

\section{PREPARATIONS}

All production data about students resided in the so-called SOSPOS database until December 2012. In Q1/2012 the migration scripts were tested for the first time. These scripts are used one-time only to migrate data from the old SOSPOS to the new HISinOne database. Log files have to be analyzed for errors and warnings, especially for non-matching data types or out-of-spec values. Since the data model for SOSPOS was open and extensible for many years, data quality checks are also required. These must focus on the changes made in the past to the SOSPOS data model by HIS (e.g. the identity model), as well as on the extensions made to this model by the university itself, e.g. chip card management, "error field" handling/definition in the student management workflow, or fee management. As HIS had released the first fee management module (FIA) in March 2012, all required tests could be carried out and successfully finished until December 2012 using various SOSPOS and HISinOne test and quality assurance systems.

As there will be HIS and Non-HIS IT systems running in 2013 and onwards that use some of the data in SOSPOS, it has been decided to regularly synchronize selected HISinOne data back to SOSPOS until the legacy systems are either phased out or their import/export filters have been ported to the HISinOne data model. Despite its regular character, this process is called remigration rather than synchronization. 
In addition, there are standard and non-standard data import/export filters that had to be tested or developed. The standard filters include MT940 and DTAUS file exchange for fee management with the bank. These had to be configured correctly and tested with the corresponding financial institutions. The non-standard filters for HISinOne include a bidirectional connector to the UDE identity management system "AUM", an interface to Uni-Assist, student loan data export for "Bafögamt", import filters for facility management data from "ConjectFM" system, and several nonstandard in-house reports.

As best practice results, the following experience has been developed: Data clearing in the SOSPOS database should be started with the first migration test runs. By analyzing the logs, various data types expected by HISinOne may require data updates procedures. Depending on the age of the SOSPOS database, issues with historical changes in the identity concept may arise that may require a one-time correction within SOSPOS itself. It is also crucial to decide about the orgunit source system in advance. Since HISinOne relies on one dedicated organizational structure, the pros and cons of using any of the various existing structures should be carefully analyzed and evaluated.

\section{ROLL-OUT}

HISinOne has a scalable three-tier architecture and currently runs on several virtualized Tomcat application servers, load balanced via the Apache mod_jk module. The HISinOne database runs on Postgres 9.1. Additional test and quality assurance systems are facilitated on demand and run on the same VMWare platform.

The regular data remigration can run as a batch job, although log file analysis and additional tests are strongly advised in the post-production phase. Technical Pre-tests and QA should not be limited to HIS systems but should include all existing target databases and platforms.

By January 2013, the new integrated software version HISinOne 4.0.1.21 had already successfully managed more than 5,500 exam registrations, 2,800 new applications with peaks of 250 per day, and more than 350 new enrollments at the UDE by the end of January 2013 (cf. Figure 1).

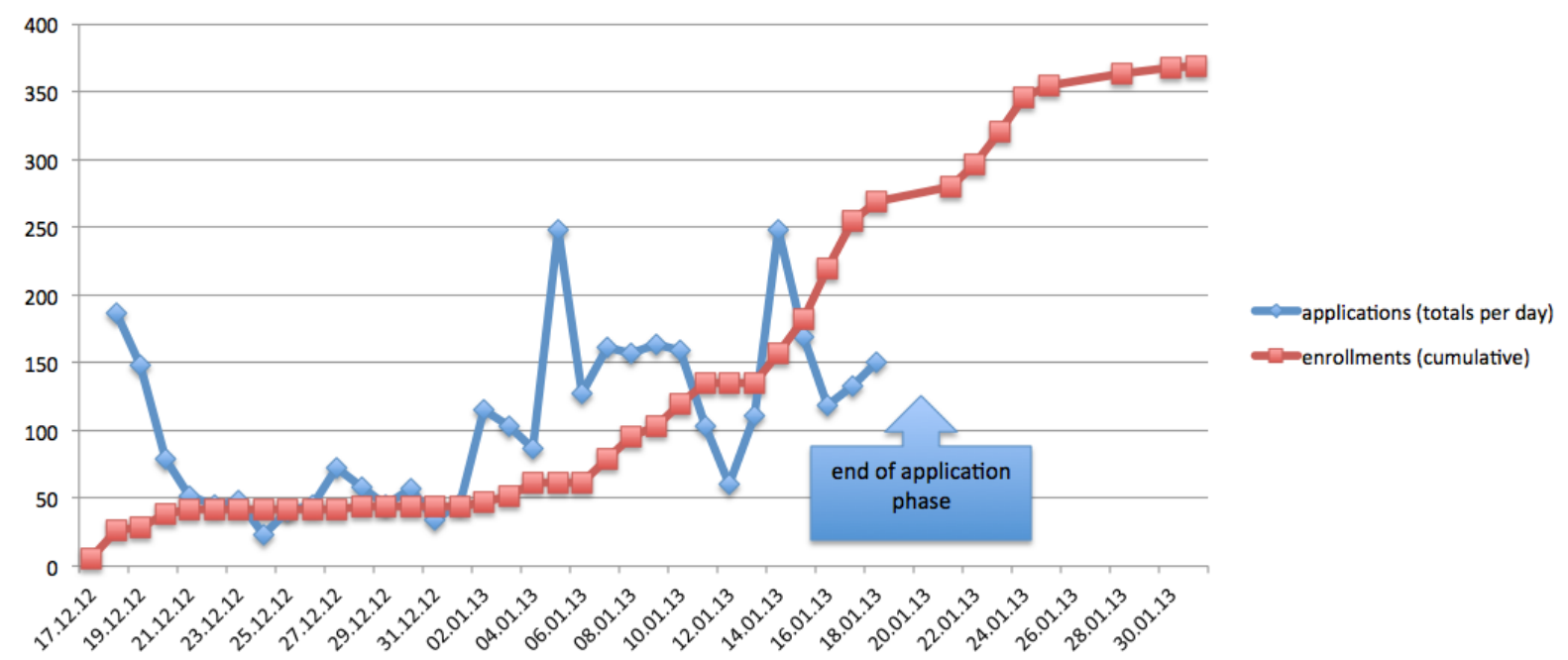

Figure 1. Amount of applications per day and cumulative enrollments, processed with an integrated HISinOne Version 4 system

\section{FUTURE WORK}

The next steps include the centralized implementation of the remaining examination regulations from the current production environment into HISinOne and the integration of lecturers.

The integration of lecturing data from HIS LSF into HISinOne EXA and the management of exams and related lectures are subject of ongoing projects. 


\section{AUTHORS' BIOGRAPHIES}

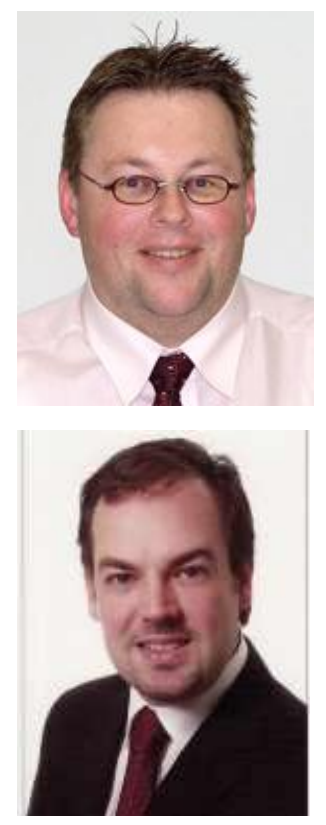

Uwe Blotevogel is the Director of the Centre of Information and Media Services (CIM) at the University of Duisburg-Essen (UDE).

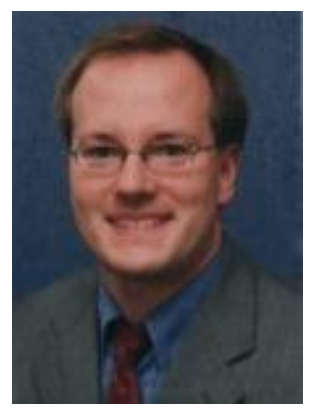

Dr Daniel Biella received his PhD degree at the UDE where he is currently working at CIM as an IT project manager. He is CIM coordinator for the implementation of HISinOne at the UDE and works in various other projects that involve web-based information systems and XML-based Metadata standards. He's also been working as a lecturer in information visualization and web-based 3D learning museums.

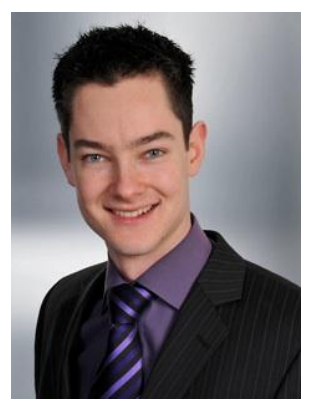

B.Sc. Malte Ney finished his studies in 2011 at FOM Hochschule für Oekonomie \& Management University of Applied Sciences. He is currently enrolled in the Masters of Science programme. Since 2011 he has been working in the Campus management as an IT system administrator at the UDE, testing and configuring the new software generation at an early adopters' site, with a focus on the student's enrollment and fee module. 